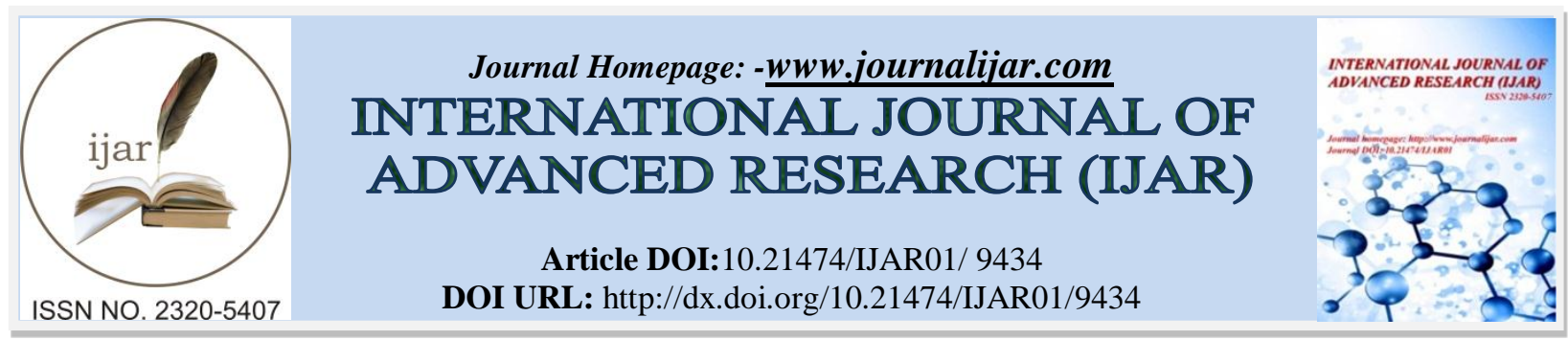

RESEARCH ARTICLE

\title{
BACTERIOLOGICAL PROFILE OF BURN PATIENTS AND ITS ANTIBIOTIC SUSCEPTIBILITY PATTERN AT A TERTIARY CARE HOSPITAL IN SOLAPUR.
}

\author{
Dr Anu Sharma ${ }^{1}$, Dr Sonal Agarwal ${ }^{2}$ and Dr N.K. Shaikh ${ }^{3}$. \\ 1. Assistant Professor, Microbiology Dr V.M.G.M.C. Solapur. \\ 2. Post Graduate Student, Microbiology, Dr V.M.G.M.C. Solapur. \\ 3. Associate Professor, Microbiology, Dr V.M.G.M.C Solapur.
}

\section{Manuscript Info}

Manuscript History

Received: 24 May 2019

Final Accepted: 26 June 2019

Published: July 2019

Key words:-

MRSA (Methicillin resistant

Staphylococcus aureus), ESBL (Extended spectrum beta lactamases),

MBL (Metallo-beta- lactamases),

Opportunistic infection.

\section{Abstract}

Introduction:Most common cause of morbidity and mortality among burn patients is infection. Infection contributes to approximately $75 \%$ of mortality among burn patients. These patients are often susceptible to opportunistic infections. The aim of the present study is to study the bacteriological profile and antibiotic susceptibility pattern of these isolates among burn patients.

Material \& method:Samples were collected from total 120 patients using sterile swab, from which 136 organisms were isolated and identified using standard procedures. Antibiotic susceptibility testing was done as per CLSI guidelines.

Result:From a total of 120 burn patient's swabs were collected. From which $115(95.83 \%)$ were culture positive and $5(4.16 \%)$ were culture negative. A total of 136 bacterial isolates were obtained from the patients. Majority of bacterial isolates were gram negative 123 $(90.44 \%)$ while gram positive isolates were $13(9.55 \%)$. Females were most commonly affected by burn accounting to $61 \%$ of cases as compared to males which were affected in $39 \%$ of cases. Most common age group affected in either sexe's was 21-30 years $(71.30 \%)$ followed by $31-40$ years $(19.13 \%)$. Burns in most of the cases was due to flame $110(96 \%)$ followed by electrocution $5(4 \%)$. Most common bacteria isolated from burn patients was Pseudomonas aeruginosa 98 (72\%) followed by Staphylococcus aureus 13 (9.555\%), Klebsiella species 20 (14.70\%), Acinetobacter species $3(2.20 \%)$ and Proteus species 2 (1.5\%). Among Staphylococcus aureus 13 (9.55\%) isolated from burn patients, high rate of MRSA 12 (92\%) were seen while MSSA 1 (8\%) case was seen. Antibiotic susceptibility pattern among gram negative bacteria showed high sensitivity to Imipenem 114 (94\%) followed by Piperacillin tazobactam 112 (71.05\%), Amikacin 110 (89.43\%), Ceftazadime $104(84.55 \%)$. Least sensitivity was seen for Cotrimoxazole (44\%) and ciprofloxacin $49 \%$ in gram negative isolates. Among gram negative isolates $12.2 \%$ isolates were ESBL producers while $4.4 \%$ isolates were MBL producers. Among gram positive isolates $100 \%$ sensitivity for seen for vancomycin \& Linezolid followed by Amikacin 12 (92.30\%), Erythromycin 8 (61.53\%),

Corresponding Author:- Dr Anu Sharma.

Address:-Assistant Professor, Microbiology Dr V.M.G.M.C. Solapur. 
clindamycin $7(54 \%)$. Least sensitivity was seen for Cefoxitin $7.7 \%$. Among gram positive bacteria $92 \%$ isolates were MRSA.

Copy Right, IJAR, 2019,. All rights reserved.

\section{Introduction:-}

Burn patients are prone to opportunistic infection and about $75 \%$ mortality among burn patient is due to infection ${ }^{1}$ .Burn sites are relatively sterile during first 24 hours thereafter colonization of wound by gram negative bacteria is common $^{2}$. Burn site is suitable niche for bacterial multiplication as larger area is involved and due to longer stay in hospital, bacteria present in the hospital environment tend to colonize the burn site and multiply rapidly to cause infection $^{3}$

Mortality among burn patients is not mainly due to osmotic shock and hypovolemia ${ }^{4}$ rather multi drug resistant bacteria colonizing the burn site may complicate the case and lead to death of patients. Most common aerobic organism isolated from burn wounds includes Staphylococcus aureus, Streptococcus pyogens, E.coli, Klebsiella species, Proteus species. Among anaerobes Bacteroides species, Peptostreptococcus, Propinobacterium species are common organism infecting burn wounds. Fungi like Aspergillus niger, Zygomycetes and Candida species are among common organisms isolated from burn wounds 5 .

The present study is done to find out aerobic bacteriological profile and antibiotic susceptibility pattern of these organisms among burn patients which will help in early management of patient and also help in decrease in mortality and morbidity among such patients.

\section{Material and method:-}

This study was conducted in department of Microbiology at a tertiary care teaching hospital in Solapur, Maharashtra. The study is a retrospective study done from May 2015 to April 2018. Permission was taken from institutional ethical committee. From a total of 120 burn patients, swabs were collected in first week between third to fifth day post admission. Two swabs were collected using aseptic precautions, one swab was used to perform gram stain and other swab was used to inoculate blood agar and Mac Conkey agar and these plates were incubated at $37^{0} \mathrm{C}$ for 18-24 hours. The isolate obtained were identified based on colony morphology, culture smear and battery of biochemical tests. Antibiotic susceptibility testing was done on Mueller Hinton agar as per CLSI guidelines 2015. ESBL production was detected using combined disc test, MBL production was detected using Modified Hodge test .

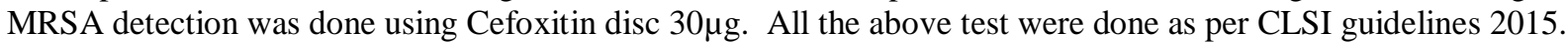

\section{Result:-}

From a total of 120 burn patient's swabs were collected. From which $115(95.83 \%)$ were culture positive and 5 $(4.16 \%)$ were culture negative. A total of 136 bacterial isolates were obtained from these patients. Majority of bacterial isolates were gram negative $123(90.44 \%)$ while gram positive isolates were $13(9.55 \%)$.

Females were most commonly affected by burn accounting to $70(61 \%)$ of cases as compared to males which were affected in 45 (39\%) of cases. Most common age group affected in either sexes was 21-30 years where $82(71.30 \%)$ cases were seen followed by 31-40 years $22(19.13 \%)$. As shown in table-1.

Table 1:-Age group and gender wise distribution in burn patients

\begin{tabular}{|l|l|l|l|}
\hline AGE GROUP (Years) & Male $(\mathbf{n}=\mathbf{4 5})$ & Female $(\mathbf{n}=70)$ & TOTAL $(\mathbf{n}=115)$ \\
\hline $10-20$ & $1(2.22 \%)$ & - & 1 \\
\hline $21-30$ & $30(66.6 \%)$ & $52(74.3 \%)$ & $82(71.30 \%)$ \\
\hline $31-40$ & $10(22.2 \%)$ & $12(17.14 \%)$ & $22(19.13 \%)$ \\
\hline $41-50$ & $4(8.8 \%)$ & $4(5.71 \%)$ & $8(7 \%)$ \\
\hline $51-60$ & - & $2(3 \%)$ & $2(2 \%)$ \\
\hline More than 60 & & - & - \\
\hline TOTAL & 45 & 70 & 115 \\
\hline
\end{tabular}

Burns in most of the cases was due to flame $110(96 \%)$ followed by electrocution $5(4 \%)$. 
Most common bacteria isolated from burn patients was Pseudomonas aeruginosa 98 (72\%) followed by Staphylococcus aureus 13 (9.55\%), Klebsiella species 20 (14.70\%), Acinetobacter species 3 (2.20\%) and Proteus species 2 (1.5\%). Among Staphylococcus aureus $13(9.55 \%)$ isolated from burn patients, high rate of MRSA 12 (92\%) were seen while MSSA $1(8 \%)$ case was seen. As shown in graph-1

Graph 1:-Distribution of bacterial isolates in burn patients

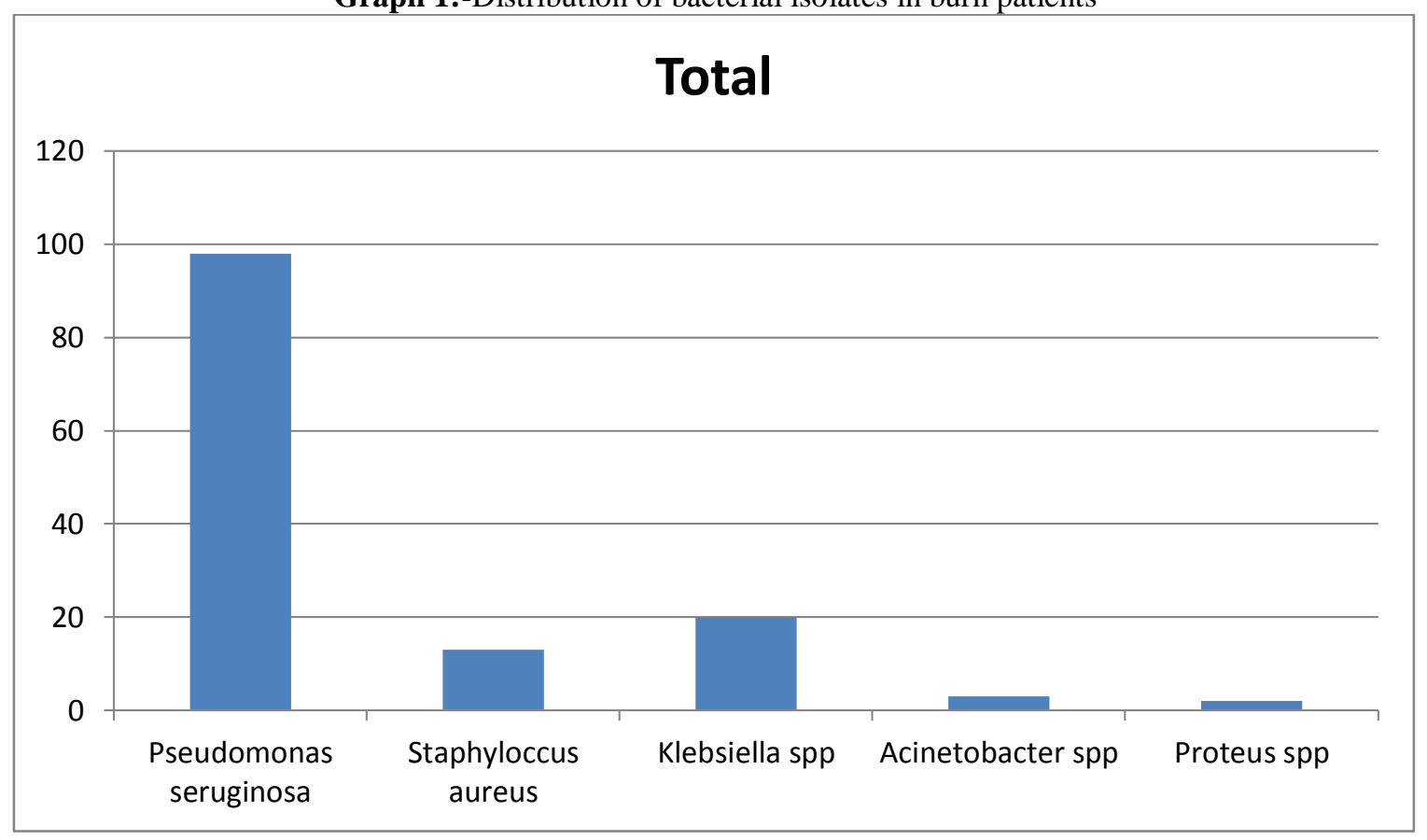

Antibiotic susceptibility pattern among gram negative bacteria showed high sensitivity to Imipenem 114 (94\%) followed by Piperacillin tazobactam 112 (71.05\%), Amikacin 110 (89.43\%), Ceftazadime 104 ( 84.55\%). Least sensitivity was seen for Cotrimoxazole (44\%) and ciprofloxacin $49 \%$ in gram negative isolates.

Among gram negative isolates $12.2 \%$ isolates were ESBL producers while $4.4 \%$ isolates were MBL producers.

Among gram positive isolates $100 \%$ sensitivity for seen for vancomycin \& Linezolid followed by Amikacin 12 (92.30\%), Erythromycin 8 (61.53\%), clindamycin 7 (54\%). Least sensitivity was seen for Cefoxitin 7.7\%. Among gram positive bacteria $92 \%$ isolates were MRSA.

\section{Discussion:-}

In the present study culture positivity rate is $95.83 \%$ which is considered as high rate. Study done by Madhumita Swain et $\mathrm{al}^{8}$ and Sharma L et $\mathrm{al}^{9}$ have also shown high culture positivity rate in burn patients. This can be attributed to the fact that disruption of skin's mechanical integrity, immuno suppression and prolonged stay in hospital all favor colonization and establishment of infection at the wound site.

In the present study females $70(61 \%)$ are more commonly affected by burns as compared to males 45 (39\%). Study done by Madhumita Swain et $\mathrm{al}^{8}$,Sharma L et al ${ }^{9}$ and Anuradha Rajput et al ${ }^{10}$ results of all these authors are in concordance to the results of the present study. This may be due to more exposure of females to flame in kitchen than in males as in Indian society females are predominantly involved in cooking and other kitchen chores and thus majority of burns are due to flame.

Most common age group affected in either sexes was 21-30 years where $82(71.30 \%)$ cases were seen followed by 31-40 years $22(19.13 \%)$. As shown in table-1.This age group have more active life style, females especially in this age group may be exposed to accidental burns in kitchen, while males may be exposed to burns due to occupation or accidental exposure to fire outside home at work place. In the present study Burns in most of the cases was due to flame $110(96 \%)$ followed by electrocution $5(4 \%)$, our studies are at par with study done by Sharma L et al ${ }^{9}$. 
Most common bacteria isolated from burn patients was Pseudomonas aeruginosa 98 (72\%) followed by Staphylococcus aureus 13 (9.55\%), Klebsiella species 20 (14.70\%), Acinetobacter species 3 (2.20\%) and Proteus species $2(1.5 \%)$. Among Staphylococcus aureus $13(9.55 \%)$ isolated from burn patients, high rate of MRSA 12 $(92 \%)$ were seen while MSSA $1(8 \%)$ case was seen. Study done by Madhumita Swain et al ${ }^{8}$, Sharma $^{2}$ et al ${ }^{9}$ and Anuradha Rajput et $\mathrm{al}^{10}$ shows finding similar our study.

Among gram positive isolates $100 \%$ sensitivity for seen for vancomycin \& Linezolid followed by Amikacin 12 (92.30\%), Erythromycin 8 (61.53\%), clindamycin 7 (54\%). Least sensitivity was seen for Cefoxitin $7.7 \%$. Among gram positive bacteria $92 \%$ isolates were MRSA.

Antibiotic susceptibility pattern among gram negative bacteria showed high sensitivity to Imipenem 114 (94\%) followed by Piperacillin tazobactam 112 (71.05\%), Amikacin 110 (89.43\%), Ceftazadime 104 ( 84.55\%). Least sensitivity was seen for Cotrimoxazole (44\%) and ciprofloxacin $49 \%$ in gram negative isolates. In the present study among gram positive and gram negative bacteria high resistance is noted for fluoroquinolones and sulphonamides, while sensitivity to other drugs used routinely is seen.

In the present study we found that among gram negative isolates $12.2 \%$ isolates were ESBL producers while $4.4 \%$ isolates were MBL producers. Among gram positive bacteria 92\% isolates were MRSA.

The pattern of organism causing infection and their antibiotic susceptibility pattern vary widely from country to country as well as from hospital to hospital and even among ICU's \& wards within one hospital. Analysis of the common organisms and their antibiogram will be helpful in knowing the local antibiotic resistance pattern, among burn patients which will guide in formulation of effective antibiotic policy and implementation of antibiotic stewardship program which will result in rational use of antimicrobial agents and prevent further drug resistance.

\section{Conclusion:-}

Early detection of isolates and their antibiotic susceptibility pattern prevent treatment failure. Steps like judicious use of antimicrobials and strict adherence of hospital infection control policies will help in better management of burn patients.

\section{Funding-}

No funding

No conflict of interest

\section{References:-}

1. Warrick A. Ames Management of major burn, update in Anaesthesia. 1999; 10 (10).

2. Clinton M, Duane R. Burn wound infection: Follow up, Infectious disease fellowship 2008.

3. Agnihotri N, Gupta V, Joshi R.M. Aerobic bacterial isolation from burn wound infections and their antibiogram- A five year study. Burns 2004;30: 241-43.

4. Donati I, Scammazo F, Gervasoni M, Mangliano A,Stankov B, Fraschini F. Infection and antibiotic therapy in 4000 burn patients treated in Milan Italy between 1976-1988. Burns 1993; 19 :345-348.

5. Mc Manus A.T., Mason A.D. Jr, Mac Manus W. F., Pruitt B. A. Jr. A decade of reduced gram negative infections and mortality, improved isolation of burned patients. Arch. Surg 1994; 129: 1306-1309.

6. Murray Patrick R, Baron Ellen Jo Jorgensen James H, Landry Marie Louise, Manual of clinical Microbiology, vol I, $8^{\text {th }}$ edition, ASM press, 2003;734-834.

7. Clinical and Laboratory Standards Institute. Performance Standards for Antimicrobial Disks Susceptibility Tests; Approved Standard. 25th informational supplement CLSI document M100-S25. Wayne, PA: CLSI; 2015.

8. Madhmita Swain, Bimoch Projna Paty, Nirupam Chayani. IOSR- IJMS. Sept 2017;16 (9): $20-23$.

9. Sharma Latika, Srivastava Harshit, Pipal Dharmendra Kumar, Dhawan Rohit, Poojan M Purohit, Bhargava Anupam. Int Surg J March 2017; 4(3) : 1019-1023.

10. Anuradha Rajput, K.P. Singh, Vijay Kumar, Rishi Saxxena, R.K. Singh. Antibacterial resistance pattern of aerobic bacteria isolates from burn patients in a tertiary care hospital. Biomedical research $2008 ; 19$ (1) :1-4. 